Classification

Physics Abstracts

42.30

\title{
Restoration of Old Motion Pictures
}

\author{
Étienne Decencière Ferrandière \\ CMM, École Nationale Supérieure des Mines de Paris, 35 rue Saint-Honoré, \\ 77305 Fontainebleau Cedex, France
}

\begin{abstract}
Résumé. - Les films anciens présentent souvent des défauts dus à des mauvaises conditions de préservation ou à des techniques de tournage rudimentaires. Les dégâts peuvent être si importants qu'ils réduisent considérablement l'intérêt ou le plaisir de les regarder. La restauration de ces films est nécessaire afin de récupérer une version proche de l'original. Une méthode de restauration automatique permettrait de traiter ces films à un coût raisonnable. Notre travail va dans ce sens. Dans cet article nous présentons quelques unes des techniques de restauration de films anciens développées dans le Centre de Morphologie Mathématique de l'École des Mines de Paris.
\end{abstract}

\begin{abstract}
Old motion pictures often present defects due to poor preservation conditions or to rudimentary shooting techniques. Damage may be so important that it considerably reduces the interest or the pleasure of watching them. Restoration of these films is necessary in order to recover a version closer to the original one. With an automatic method, restoration would be affordable for most pictures. Our work goes in that direction. In this paper some techniques of motion picture restoration developed in the Centre for Mathematical Morphology of the Paris School of Mines are presented.
\end{abstract}

\section{Introduction}

Old motion pictures often present defects due to poor preservation conditions or to rudimentary shooting techniques. Restoration of these films using manual techniques is very expensive because of the large number of images to be repaired: with 24 frames per second, one has $24 \times 60 \times 60=$ 86400 images per hour of film. An automatic technique that would remove most typical defects would be more affordable and faster. Thus thousands of old motion pictures, that are today in too bad shape to be correctly appreciated, could be restored.

There are many kinds of defects in motion pictures. In the first section of this paper the defects we will deal with are described, i.e. local defects. In the following sections the general structure of the restoration procedure and some detection and data recovery techniques based on morphological tools are presented. 


\section{Local Defects}

In this paper we will show how to restore local defects using morphological tools (we call "local" those defects that only affect a relatively small part of each frame). Two sorts of local defects can be distinguished. Local Time-Correlated (LTC) defects are local defects that appear in almost the same position in neighbouring frames; white vertical scratches are one example. Local Non-Time-Correlated (LNTC) defects are such that their position on one frame does not give any information about their position on the other frames. White dots produced by electrostatic electricity and crackles of the varnish layer of the film belong to this category.

Mathematical morphology tools [1] are well adapted for this kind of problem because their geometrical properties are very useful for object recognition, not only in the 2D domain, but also in the $3 \mathrm{D}$ one.

\section{Structure of the Restoration Method}

In this paper the problem of the restoration of local defects, time-correlated and non-time-correlated, will be treated. The general structure of the method is the same in both cases. It is based on the algorithm that is presented in [2].

First, there is a detection phase, whose purpose is to detect as precisely as possible the defects. The result of this phase will be a binary image where the "white" pixels will correspond to the defects.

The second phase is the data recovery. The defects destroy the information that was in their location so one has to interpolate it from the data that are left in the sequence. It is important to note that only the pixels that were marked as defects at the end of the detection phase will be modified. The others will be left unchanged.

The algorithm will work with a sequence of frames $F(t)$ that can be treated as a $3 \mathrm{D}$ image, where the third axis is time. But the entire sequence cannot be simultaneously treated because of memory limitations, so a recursive method has been adopted. Suppose that the current time is $t_{0}$. One takes frames $F\left(t_{0}-N\right), \ldots, F\left(t_{0}-1\right), F\left(t_{0}\right), F\left(t_{0}+1\right), \ldots, F\left(t_{0}+N\right)$ and builds with them a 3D image I. At each iteration the algorithm works on this image. $N$ border frames have been added at both ends of the 3D image $I$ in order to avoid border problems with temporal filtering. $N$ will depend on the size of the temporal structuring element that will be used.

Example. - In this paper an extract from a documentary by the Lumière brothers has been used as test sequence. There are 16 frames per second. The resolution of the digitized picture is $1200 \times 900$, but only the central part of the picture, of size $512 \times 512$, has been used for practical reasons. In Figure 2a 5 images of the test sequence are shown. They were damaged by dark crackles and white dots, which are both LNTC defects, and by white vertical scratches, which are LTC defects. The results of our restoration algorithm will be illustrated with the third frame of this sequence, which is more visible on the top of Figure 6. As one can see, on one hand the image quality is poor; it is very noisy and contrast is low, this makes the restoration procedure more complicated. On the other hand, the movement is simple; it is basically a forward movement added to the vibrations of the camera.

\section{Detection}

4.1 Frame per Frame Approach. - The first method exploits the first characteristic of local defects: their shape. This is a classical pattern recognition problem. However the shape of defects is often difficult to model, and it is easy to mistake true objects for defects. 


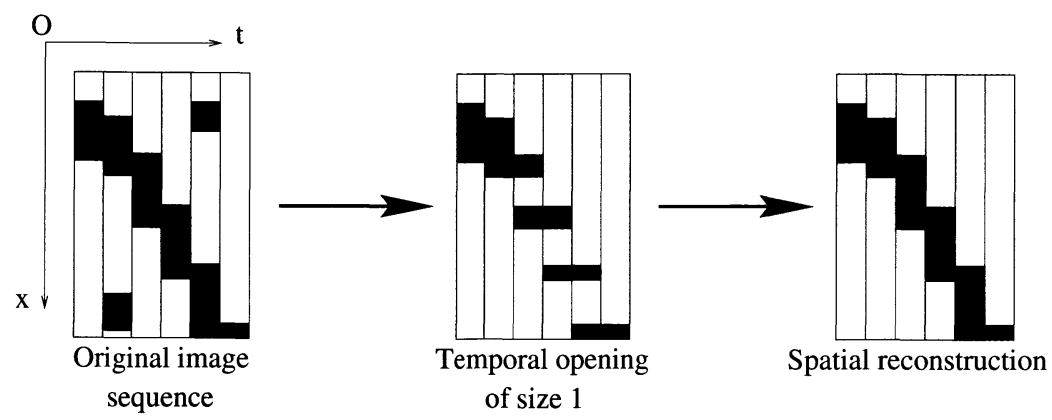

Fig. 1. - Example of application of the temporal opening followed by a spatial reconstruction on a binary image sequence.

a)



b)

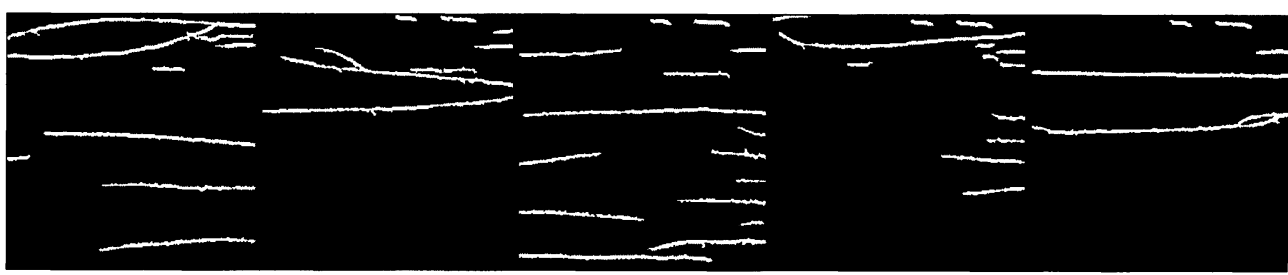

c)

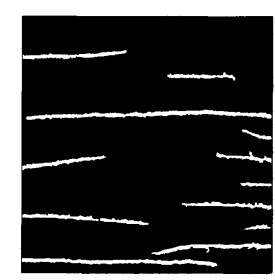

Fig. 2. - Detection of crackles. a) Old motion picture with some typical defects. b) Frame per frame detection of crackles. c) Selection of crackles that are thin along the time axis.

Consequently, at the end of this phase one shall obtain a binary image marking defects and some scene objects whose geometrical spatial properties are similar to those of the defects that are to be detected. How the difference between true defects and false detections can be made?

4.2 3D ApproACH. - The 3D approach takes advantage of the temporal characteristics of local defects. As such, it will be different for LTC and LNTC defects. In both cases it will take as input the binary image produced by the $2 \mathrm{D}$ detection phase and use the temporal properties of the defects in order to keep only the true defects of the image and throw away the false detections. 
Let us consider an image sequence as a 3D image, where the third axis is time. LTC defects tend to appear in the same position in several consecutive frames, so they will appear as long objects along the time axis. That is, they will be similar to still or slow moving scene objects.

Moreover, most of the time, LNTC defects will have a small time dimension (they appear at the same place only in one frame), and, as such, they will be similar to fast moving scene objects (an object of the scene is said to be fast moving when its movement is fast enough to disconnect it along the time axis).

In most natural scenes, slow moving objects are much more common than fast moving objects. As a consequence, temporal filtering will be more useful to detect LNTC defects than LTC defects. Here it will be applied only to the detection of LNTC defects.

As said previously, LNTC defects are thin along the time axis, so an opening [1] with a structuring element parallel to the time axis should erase them. In order to avoid any modification of the contours of the objects that are not completely erased by the opening, a geodesic spatial reconstruction [3] will be added. The effect of these operations is illustrated in Figure 1. Thin objects along the time axis have been completely erased, whereas other objects have been left unchanged.

What length must be chosen for the temporal structuring element? It depends on the density of the defects: the higher the defect probability, the longer the structuring element should be. In our example a size 2 linear structuring element was taken: therefore $N=2$ border frames at each end of $I$ were used.

Note that the longer the temporal structuring element, the more scene objects will be detected as being fast moving. In fact, to avoid the problem of fast moving objects, a motion compensation phase is needed. But motion compensation is a difficult problem, and the difficulty is even more important in the framework of image restoration because of the bad shape of the images. Therefore, in this study a motion compensation phase is not used; the connectivity along the time was the only criterion used to distinguish between defects and scene objects.

4.3 ExAmPLE. - Our detection method, applied to the case of dark crackles, which are LNTC defects, was applied to our test sequence 2(a). Image (b) shows the resulting binary mask after the frame per frame detection. Observe that the shadow of the roofs has been detected because it is similar to the objects we are looking for. But, when one keeps only thin-time objects, most of these false detections disappear (see Fig. 2c). In fact the main problem involves the objects that are at the edge of the detection field, and as such, are detected only in some frames, producing this way a flicker effect. In the example there is such a case: it is the shadow of the roof of the central building. It has been considered as a defect in two frames of the sequence, which is not enough to reject it as a false defect. It is very difficult to avoid this kind of mistake; one can only hope that the data recovery phase will reconstruct the false defect in a correct way.

\section{Data Recovery}

As the defects have been identified, the missing information must now be restored. There are again two approaches.

5.1 Frame Per Frame Data Recovery. - The 2D approach is based on the hypothesis that there are enough data around the missing region to restore the information inside this region. So, the idea is to interpolate structures or textures surrounding the missing area in order to restore it. Two methods have been tested.

Dilations of the grey levels around the missing area. - This method gives good results only in the case of very thin defects. Otherwise the resulting texture has a very poor visual quality. An example of restoration of crackles using this method is presented in Figure 3. 

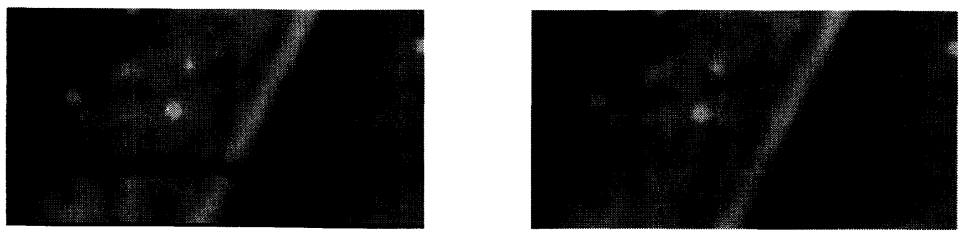

Fig. 3. - Example of restoration of a crackle using dilation.
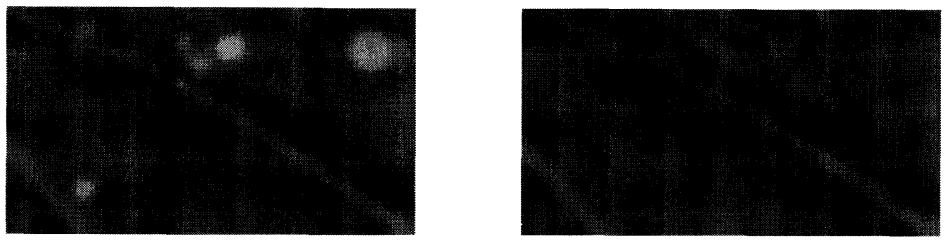

Fig. 4. - Restoration of white dots using extrapolation of Fourier coefficients.
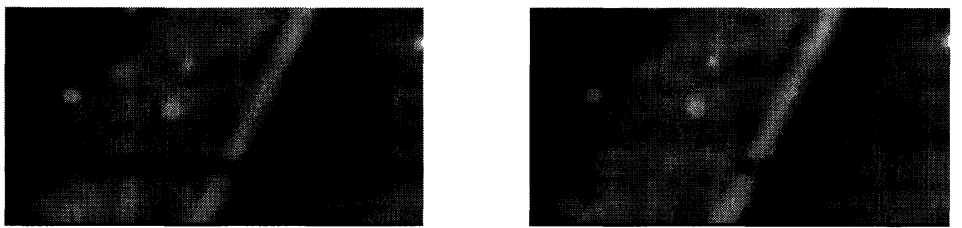

Fig. 5. - Example of restoration of a crackle using a temporal closing.
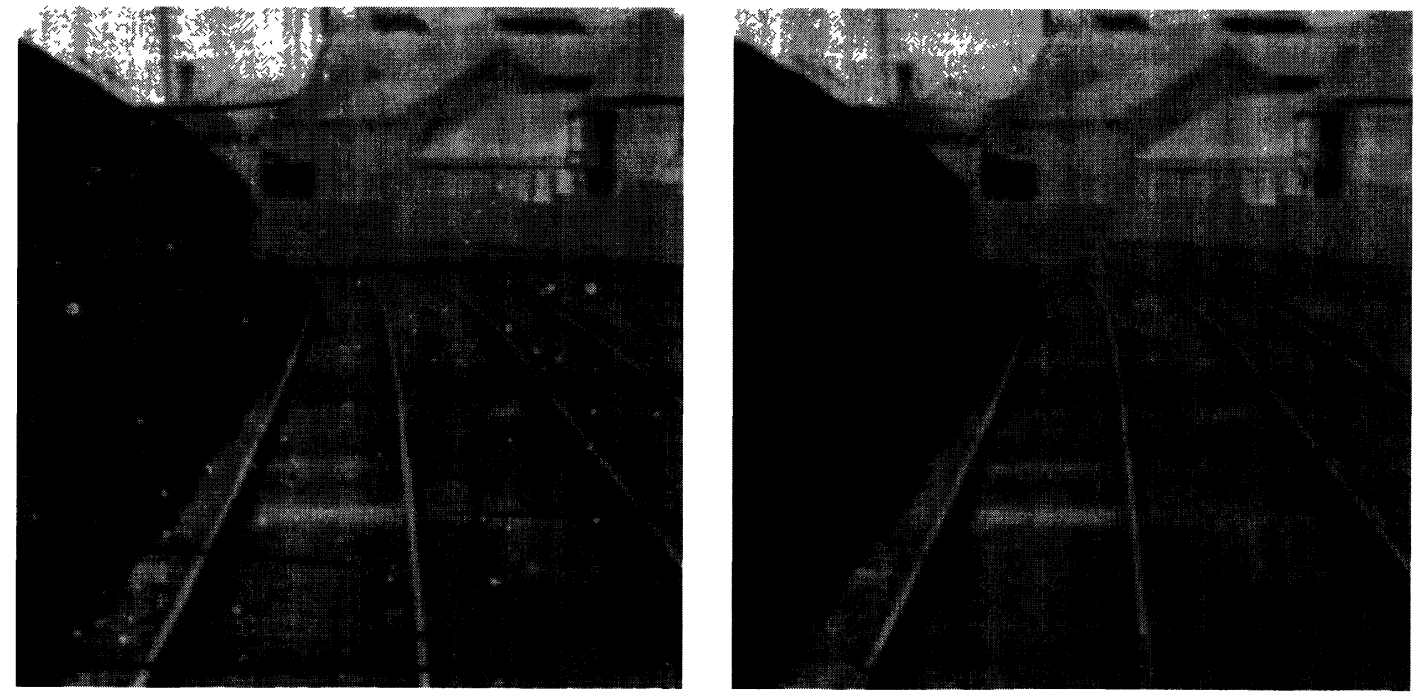

a)

b)

Fig. 6. - Restoration of crackles, white dots and white scratches on our test image. a) Image before restoration. b) Image after restoration. 
Extrapolation inside the missing area of Fourier coefficients calculated around it. - This method, developed by Van Droogenbroeck [4], gives good results for textures, but does not respect the structures around the defect. An example of restoration of white dots using this method is presented in Figure 4.

The 2D approach may be sufficient in the case of small defects or in the case of defects in smooth regions. In other cases the information around the defects is not enough to restore the missing data, especially when an important object has disappeared from the scene because of the damage. The only solution is then to use the information of the other frames.

5.2 Time Interpolation. - The second approach takes advantage of the image sequence. Due to the fact that LNTC defects are not correlated in time, the information that was lost in a frame will generally be present in the next or previous compensated frame. Therefore, the objective is to find the region in the neighboring frames that corresponds to the missing area. Again, this method will not work very well for LTC defects because in most cases the defect will have destroyed the information in the same region of the scene in the neighbouring frames. It has been applied only to LNTC defects.

Of course, if there is no movement in the scene, the information that was lost in one frame should be similar to the information in the same position in the neighbouring frames (as long as it has not been destroyed by a defect too!). In this case, a simple time interpolation is enough to recover the lost information. For example, a closing with a linear structuring element of size 2 (same length as the one used for the temporal detection) parallel to the time axis can be sufficient. When there is movement, one can use a motion compensation algorithm, with the difficulties that this involves, or simply restrict himself to the spatial interpolation. An example of this technique is shown in Figure 5.

\section{Results and Conclusion}

An example of restoration of dark crackles, white dots and white scratches is shown in Figure 6. As can be seen, we succeeded in eliminating most of them. The roof of the central building, which had been mistaken for a crackle in the detection phase, has been partially erased. However, the quality of the restored image is much better than the original one. It is important to note that the regions that were not detected as defects have not been modified.

In this paper some methods of motion picture restoration for local time-correlated and local non-time-correlated defects based on morphological tools have been presented. Note that the structure of the restoration method is very general and can be easily applied to other local defects. This prospective work has given very good results: the spatial and temporal properties of the defects have been successfully used to detect them and different interpolation tools for the data recovery phase have been tested.

Future research will deal with the restoration of global defects and with the development of motion compensation algorithms able to work in a noisy environment.

\section{References}

[1] Serra J., Image analysis and mathematical morphology (Academic Press, 1982).

[2] Decencière Ferrandière E., Motion picture restoration using morphological tools, in "International Symposium on Mathematical Morphology (ISMM'96)”, Atlanta (GA), USA (May 1996).

[3] Vincent L., Morphological gray scale reconstruction in image analysis: Applications and efficient algorithms, IEEE Trans. on Image Proc. 2 (1993) 176-201.

[4] Van Droogenbroeck M., Traitement d'images numériques au moyen d'algorithmes utilisant la morphologie mathématique et la notion d'objet: application au codage, Thèse de Doctorat de l'École des Mines de Paris (May 1996). 